\title{
Cycle time optimization of deterministic timed weighted marked graphs by transformation
}

\author{
Zhou He, Zhiwu Li, Alessandro Giua,
}

\begin{abstract}
Timed marked graphs, a special class of Petri nets, are extensively used to model and analyze cyclic manufacturing systems. Weighted marked graphs are convenient to model automated production systems such as robotic work cells or embedded systems and reduce the size of the model. The main problem for designers is to find a trade-off between minimizing the cost of the resources and maximizing the system's throughput (also called cycle time). It is possible to apply analytical techniques for the cycle time optimization problem of such systems. The problem consists in finding an initial marking to minimize the cycle time (i.e., maximize the throughput) while the weighted sum of tokens in places is less than or equal to a given value. We transform a weighted marked graph into several equivalent marked graphs and formulate a mixed integer linear programming model to solve this problem. Moreover, several techniques are proposed to reduce the complexity of the proposed method. We show that the proposed method can always find an optimal solution.
\end{abstract}

Published as:

Zhou He, Zhiwu Li, and A. Giua. "Cycle time optimization of deterministic timed weighted marked graphs by transformation,” IEEE Trans. on Control Systems Technology, 2016. To appear. DOI: 10.1109/TCST.2016.2613967

Zhou He is with the School of Electro-Mechanical Engineering, Xidian University, Xi'an 710071, China, and also with Aix Marseille Univ, Université de Toulon, CNRS, ENSAM, LSIS, Marseille, France. e-mail: zhouhe@stu.xidian.edu.cn

Zhiwu Li is with the Institute of Systems Engineering, Macau University of Science and Technology, Taipa, Macau, and also with School of Electro-Mechanical Engineering, Xidian University, Xi’an 710071, China. e-mail: zhwli@xidian.edu.cn

Alessandro Giua is with Aix Marseille Univ, Université de Toulon, CNRS, ENSAM, LSIS, Marseille, France, and also with DIEE, University of Cagliari, Cagliari 09124, Italy. email: alessandro.giua@univ-amu.fr, giua@diee.unica.it 


\section{INTRODUCTION}

Petri nets (PNs) have found their extensive applications to the supervisory control [1], [2], [13], [14], scheduling [11], and analysis [5], [9] of discrete event systems including flexible manufacturing systems. In real-world systems, activities do not take place instantaneously. Every activity in a system has a time duration which is different from zero. Timed PNs are well known as efficient tools for modeling discrete event systems and representing their dynamic behaviors. In this paper, we study a particular class of timed PNs called timed weighted marked graphs (TWMGs). The main feature of this class of nets is that each place has only one input and one output transition and arcs have a positive integer weight. Moreover, the firing delay of each transition is deterministic.

Timed weighted marked graphs and timed marked graphs (TMGs) find wide applications in manufacturing. They can model complex assemble lines and solve cyclic scheduling problems. Workshop operations and products are usually modeled by transitions and tokens, respectively. Between two successive transformations, semi-finished products have to be stored or moved from a workshop to another. The amount of products, also called Work In Process (WIP), that have to be stored or moved may have economical consequences. Therefore, the main problem for designers is to find a proper schedule of WIP that allows the system to reach a given productivity while the amount of WIP is the smallest.

Some results have been developed for this class of PNs. For instance, Teruel et al. [3] and Chrzastowski-Wachtel and Raczunas [4] proposed several techniques for the analysis of WMGs. Campos et al. [6] developed methods to compute the cycle time of TMGs for a given initial marking. Munier [7] proposed a method to transform a WMG into an MG under single server semantics hypothesis and Nakamura and Silva [8] discussed the same problem under infinite server semantics hypothesis. Benabid-Najjar et al. [10] studied the periodic schedules of bounded TWMGs and presented polynomial algorithms to check the existence of periodic schedules. Giua et al. [12] dealt with the firing rate optimization of cyclic timed event graphs by token allocations and proposed a mixed integer linear programming problem (ILPP) to compute an optimal solution.

Other approaches based on the so-called tropical algebras, such as $(\max ,+)$ or $(\min ,+)$, have been widely used to describe the behavior and analyse the performance of TWMGs [15]-[19]. The weights on the arcs of a TWMG correspond to non-linear models in tropical algebra. Thus, a linearization method was proposed in [20] when each elementary circuit contains at least one unitary transition (i.e., a transition for which its corresponding elementary T-semiflow component is equal to one). This method increases the number of transitions. Inspired by this work, some linearization methods without increasing the number of transitions were proposed in [21], [22]. The obtained $(\min ,+)$ linear model allows to evaluate the performance of the model.

However, in the literature, few works deal with the optimization problem of TWMGs. Benazouz et al. [28] developed an algorithm to minimize the overall buffer capacities with throughput constraint for TWMGs. Sauer [29] proposed a heuristic solution based on an iterative process to solve the marking optimization problem of TWMGs.

The marking optimization problem of TWMGs consists in reaching a desired cycle time while minimizing a linear combination of markings in places. He et al. [30], [31] presented a novel heuristic method to deal with the marking 
optimization problem which was shown to be more effective than that of Sauer [29]. Nevertheless, the presented solutions are heuristic and the optimality is not ensured. Thus, the problem of finding an optimal solution for marking optimization of TWMGs is still open.

In this paper, we study the cycle time optimization problem of a TWMG, an issue that to the best of our knowledge has not been addressed in the literature. The problem consists in finding an initial marking to minimize the cycle time while the weighted sum of tokens in places is less than or equal to a given value. This problem has a practical relevance in many applications. As an example, for a manufacturing system operated with a periodic scheduling the cycle time is the inverse of the throughput. Thus if the cycle time is minimized, the throughput is maximized. In addition, in a PN model of a manufacturing system, tokens in the net represent resources allocated to it such as machines, transportation devices, buffer slots, etc. Thus a bound on the weighted sum of tokens in the net describes a limited availability of resources or equivalently a limited budget to acquire them. We transform a TWMG into several equivalent TMGs and formulate a mixed ILPP solution following the approach of Giua et al. [12] to compute a proper initial marking. The conversion of the obtained marking for the equivalent TMG into a corresponding marking of the TWMG is presented. Moreover, several measures are taken to improve the algorithm to reduce the computation cost. We show that the proposed method can always find an optimal solution.

The resource optimization problem based on PNs has been extensively studied in the literature. For instance, Wang and Zeng [23] studied a time PN model of workflows constrained by resources. They proposed a method to verify the risks and found the best implementation case by assuming that all required resources have been prepared well before the start of the activity. Hee et al. [24] and Li and Reveliotis [25] presented some methods to compute optimal resource allocation in stochastic PNs. Chen et al. [26] developed a new PN model called resource assignment PN to compute the time needed to execute each project under the described scenarios. Rodriguez et al. [27] proposed a heuristic method to solve the resources optimization problem for process systems with shared resources under the assumption that the considered PNs are live. By contrast to the aforementioned works, we are interested in resource optimization for TWMGs which are conflict free nets, i.e., there exist no shared resources. Moreover, the TWMGs are not initially assumed to be live, i.e., we need to find a live resources assignment policy which maximizes the throughput of the system.

The main contributions of the present paper can be summarized as follows:

(1) Periodicity of transformation of TWMGs into TMGs is formalized and the initial marking of a TWMG is partitioned into several subsets with regard to the periodicity.

(2) Transformation of the cycle time optimization problem of TWMGs into the cycle time optimization problem of TMGs is developed.

(3) A mixed ILPP combined with the results reported in [12] is presented to deal with the cycle time optimization problem of TWMGs. We show that the proposed method can always find an optimal solution.

(4) Some techniques are introduced in Section V-A to reduce the computational burden of computing the solution.

(5) A more general cycle time optimization problem is discussed to maximize the throughput and minimize the cost of the resources. 
(6) Experimental studies are reported in Section VI to validate the efficiency of the proposed approaches.

Some of these results were presented in a preliminary form in [32]. In this extended manuscript, detailed proofs of Propositions 2, 5, 6, 7 and Property 1 are given. Moreover, the proposed algorithms are formalized and items (4)-(6) of the main contributions are originally presented.

This paper is structured as follows. In the following section, we briefly recall some basic concepts and the main properties of TWMGs. In Section III, we present the problem statement. In Section IV, we propose an analytical method for the cycle time optimization problem based on the work in [12]. Several measures are taken to improve the algorithm to reduce the computation cost in Section V-A. Moreover, we study a more general optimization problem. Some experimental results are presented in Section VI. Conclusions and future work are finally drawn in Section VII.

\section{BACKGROUND}

\section{A. Generalities}

We assume that the reader is familiar with the structure, firing rules, and basic properties of PNs (see [3], [33], and [34]). In this section, we will recall the formalism used in this paper. A PN is a structure $N=(P, T$, Pre, Post $)$, where $P$ is a set of $n$ places; $T$ is a set of $m$ transitions; Pre $: P \times T \rightarrow \mathbb{N}$ and Post $: P \times T \rightarrow \mathbb{N}$ are the preand post-incidence functions that specify the arcs; $\boldsymbol{C}=$ Post-Pre is the incidence matrix, where $\mathbb{N}$ is a set of non-negative integers.

A vector $\boldsymbol{x}=\left(x_{1}, x_{2}, \ldots, x_{m}\right)^{T} \in \mathbb{N}^{|T|}$ such that $\boldsymbol{x} \neq \mathbf{0}$ and $\boldsymbol{C} \cdot \boldsymbol{x}=\mathbf{0}$ is a T-semiflow. A vector $\boldsymbol{y}=$ $\left(y_{1}, y_{2}, \ldots, y_{n}\right)^{T} \in \mathbb{N}^{|P|}$ such that $\boldsymbol{y} \neq \mathbf{0}$ and $\boldsymbol{y}^{T} \cdot \boldsymbol{C}=\mathbf{0}$ is a P-semiflow. The supports of a T-semiflow and a P-semiflow are defined by $\|\boldsymbol{x}\|=\left\{t_{i} \in T \mid x_{i}>0\right\}$ and $\|\boldsymbol{y}\|=\left\{p_{i} \in P \mid y_{i}>0\right\}$, respectively. A minimal T-semiflow ${ }^{1}$ (resp. P-semiflow) is a T-semiflow $\|\boldsymbol{x}\|$ (resp. P-semiflow $\|\boldsymbol{y}\|$ ) whose support is not a superset of the support of any other T-semiflow (resp. P-semiflow), and whose components are mutually prime.

A marking is a vector $M: P \rightarrow \mathbb{N}$ that assigns to each place of a PN a non-negative integer number of tokens; we denote the marking of place $p$ as $M(p)$. A PN system or net system $\left\langle N, \boldsymbol{M}_{\mathbf{0}}\right\rangle$ is a net $N$ with an initial marking $\boldsymbol{M}_{\mathbf{0}}$. A transition $t$ is enabled at $\boldsymbol{M}$ if $\boldsymbol{M} \geq \operatorname{Pre}(\cdot, t)$ and an enabled transition $t$ may fire yielding a new marking $M^{\prime}$ with

$$
\boldsymbol{M}^{\prime}=\boldsymbol{M}+\boldsymbol{C}(\cdot, t)
$$

where $\operatorname{Pre}(\cdot, t)$ (resp. $C(\cdot, t)$ ) denotes the column of the matrix Pre (resp. $C$ ) associated with transition $t$. Marking $\boldsymbol{M}^{\prime \prime}$ is said to be reachable from $\boldsymbol{M}$ if there exist a sequence of transitions $\sigma=t_{0} t_{1} \ldots t_{n}$ and markings $\boldsymbol{M}_{1}, \boldsymbol{M}_{2}, \ldots$, and $\boldsymbol{M}_{n}$ such that $\boldsymbol{M}\left[t_{0}\right\rangle \boldsymbol{M}_{1}\left[t_{1}\right\rangle \boldsymbol{M}_{2} \ldots \boldsymbol{M}_{n}\left[t_{n}\right\rangle \boldsymbol{M}^{\prime \prime}$ holds. The set of markings reachable from $\boldsymbol{M}_{0}$ in $\left\langle N, \boldsymbol{M}_{0}\right\rangle$ is called the reachability set of the PN system $\left\langle N, \boldsymbol{M}_{0}\right\rangle$ and denoted by $R\left(N, \boldsymbol{M}_{0}\right)$.

A PN is said to be ordinary when all its arc weights are 1's. A marked graph (also called an event graph) is an ordinary PN such that each place has only one input transition and one output transition. A weighted marked graph

\footnotetext{
${ }^{1}$ This is also called a minimal and minimal support semiflow in some references. For the sake of simplicity, we call it a minimal semiflow.
} 
(also called a weighted event graph) is a net such that each place has only one input transition and one output transition but may not be ordinary, i.e., the weight associated with each arc is a positive integer number.

A deterministic timed PN is a pair $N^{\delta}=(N, \delta)$, where $N=(P, T$, Pre, Post $)$ is a PN net, and $\delta: T \rightarrow \mathbb{Q}^{+}$, called firing delay, assigns a non-negative rational firing duration to each transition. It is easy to see that without loss of generality we can equivalently represent any rational time durations in a given net by natural numbers. Therefore, in the rest of this paper, we assume the domain of every firing delay function to be $\mathbb{N}$. A transition with a firing delay equal to 0 is said to be immediate. A clock $\theta_{i}(t)$ associated with transition $t$ represents the residual time to fire $t$ at marking $\boldsymbol{M}_{i}$. If a transition $t$ is not enabled at marking $\boldsymbol{M}_{i}$, it is usually assumed that $\theta_{i}(t)=+\infty$. We denote the clock vector $\boldsymbol{\theta}_{\boldsymbol{i}}=\left(\theta_{i}\left(t_{1}\right), \theta_{i}\left(t_{2}\right), \ldots, \theta_{i}\left(t_{m}\right)\right)$.

We denote the state of a TWMG as $[\boldsymbol{M} ; \boldsymbol{\theta}]$. Thus the evolution of a deterministic TWMG will be described by the marking $\boldsymbol{M}_{i}$, the clock vector $\boldsymbol{\theta}_{\boldsymbol{i}}$ and the time instant $\tau_{i}$ at which marking $\boldsymbol{M}_{i}$ is reached (for $i=1,2, \ldots, n$ ). We initialize $\tau_{0}=0$ for the initial marking $\boldsymbol{M}_{0}$.

When a transition $t$ becomes enabled, it cannot fire before the time $\delta(t)$ has elapsed. Under the as soon as possible (ASAP) execution policy, a transition $t$ will fire exactly after it has been enabled for a time $\delta(t)$. In this paper, we deal with TWMGs: they are conflict-free nets, and thus the firing of a transition $t$ does not disable any other transition [34]. In the ASAP execution, from state $\left[\boldsymbol{M}_{i} ; \boldsymbol{\theta}_{\boldsymbol{i}}\right]$ all transitions that have a minimal value of the clock can be fired simultaneously after a time $\phi_{i}=\min _{t \in T} \theta_{i}(t)$ and the TWMG reaches a new state $\left[\boldsymbol{M}_{i+1} ; \boldsymbol{\theta}_{\boldsymbol{i + 1}}\right]$. The marking will change as shown in Eq. (1) and the clock will be updated by the following equations:

$$
\begin{aligned}
& \theta_{0}(t)= \begin{cases}\delta(t), & \text { if } \boldsymbol{M}_{0}[t\rangle \\
+\infty, & \text { if } \neg \boldsymbol{M}_{0}[t\rangle\end{cases} \\
& \theta_{i}(t)= \begin{cases}\delta(t), & \text { if } \neg \boldsymbol{M}_{i-1}[t\rangle \text { and } \boldsymbol{M}_{i}[t\rangle \\
+\infty, & \text { or t fired at } \boldsymbol{M}_{i-1} \text { and } \boldsymbol{M}_{i}[t\rangle \\
\theta_{i-1}(t)-\phi_{i-1}, & \text { if } \neg \boldsymbol{M}_{i}[t\rangle\end{cases}
\end{aligned}
$$

where $\neg \boldsymbol{M}[t\rangle$ means that $t$ is not enabled at marking $\boldsymbol{M}$. In this paper, we consider a single server semantics, i.e., we assume that each transition can fire only once at each time instant even if its enabling degree is greater than one (see [29]).

A net is strongly connected if there exists a directed path from any node in $P \cup T$ to every other node. Let us define an elementary circuit $\gamma$ (or elementary cycle) of a net as a directed path that goes from one node back to the same node without passing twice on the same node. In a strongly connected net, it is easy to show that each node belongs to an elementary circuit, and thus the name cyclic nets is also used to denote this class.

Given a place $p$ of a WMG, let $t_{i n(p)}$ (resp., $\left.t_{\text {out }(p)}\right)$ be its unique input (resp., output) transition as shown in Fig. 1. We denote $w(p)=\operatorname{Post}\left(p, t_{i n(p)}\right)$ the weight of its input arc and $v(p)=\operatorname{Pre}\left(p, t_{\text {out }(p)}\right)$ the weight of its output arc. We denote $g c d_{p}$ the greatest common divisor of the integers $w(p)$ and $v(p)$. 


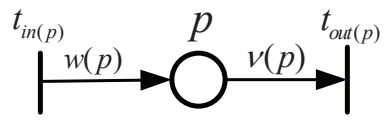

Fig. 1. A place $p$ between two transitions $t_{i n(p)}$ and $t_{o u t(p)}$.

Definition 1: Every elementary circuit $\gamma$ of a WMG is neutral, if the following condition holds.

$$
\prod_{p \in \gamma} \frac{v(p)}{w(p)}=1
$$

In other words, in a neutral circuit the product of the weights of all pre-arcs is equal to that of all post-arcs. This means that if the circuit initially contains enough tokens, it is possible to fire all transitions along the path returning to the same initial marking. It is well known that a WMG whose circuits are all neutral has a unique minimal T-semiflow $\boldsymbol{x}$ and it contains all transitions in its support [3].

Proposition 1: (Benabid-Najjar et al. [10]) A strongly connected WMG in which all circuits are neutral is bounded, i.e., there exists an integer $B$ such that the marking of any place $p$ is not greater than $B$ at any reachable marking.

In this paper, we limit our study to strongly connected WMGs in which all circuits are neutral.

\section{B. Cycle time of a TWMG}

The cycle time $\chi\left(\boldsymbol{M}_{0}\right)$ of a TWMG system $\left\langle N, \boldsymbol{M}_{\mathbf{0}}\right\rangle$ is the average time to fire once the minimal T-semiflow under the ASAP operational model. For deterministic TWMGs, the following limit exists:

$$
\lim _{\tau \rightarrow \infty} \frac{\vec{\sigma}_{\tau}}{\tau}=\vec{\sigma}^{*}<\vec{\infty}
$$

where the vector $\vec{\sigma}_{\tau}$ represents the firing vector from time 0 to time $\tau$ and the constant vector $\vec{\sigma}^{*}$ is called the limit firing vector. We denote $\vec{\sigma}^{*}\left(t_{i}\right)$ the average number of firing $t_{i}$ per time unit.

Definition 2: Let $t_{i} \in T$ be an arbitrary transition of a TWMG $\left\langle N, \boldsymbol{M}_{0}\right\rangle$ with the minimal T-semiflow $\boldsymbol{x}$. The cycle time of the TWMG is

$$
\chi\left(M_{0}\right)=\frac{x_{i}}{\vec{\sigma}^{*}\left(t_{i}\right)} .
$$

Note that the value of the cycle time does not depend on the considered transition. In [35], [36], [37], the authors proved that the ASAP execution of a live and strongly connected TMG with integer delays is ultimately repetitive following a periodical pattern of period $\Psi$. In the case of TWMGs, the ASAP execution is also ultimately periodic. The number of firings of transition $t_{i}$ within the periodical pattern is $f_{i}$. The cycle time of the TWMG $\left\langle N, M_{0}\right\rangle$ is thus equal to

$$
\chi\left(\boldsymbol{M}_{0}\right)=x_{i} \cdot \frac{\Psi}{f_{i}}
$$


From [6] and [38], a lower bound for the cycle time of a live and bounded TWMG system can be computed analytically by solving an LPP. Chao et al. [39] proposed a method to compute the cycle time of a TWMG but under restrictive conditions at initial marking. In the general case, one may resort to simulation to compute the cycle time of a TWMG: as an example in [30] we used the PN tool HYPENS [40].

\section{Transformation of WMGs}

One way to analytically compute the cycle time of a TWMG is to convert it into an equivalent TMG. In fact, Munier [7] showed that a TWMG system $\langle N, \boldsymbol{M}\rangle$ can be transformed into an equivalent TMG system $\langle\hat{N}, \hat{\boldsymbol{M}}\rangle$ which describes the same precedence constraints on the firing of transitions. This implies that the cycle time ${ }^{2}$ of the two systems is identical, i.e.,

$$
\chi(\boldsymbol{M})=\hat{\chi}(\hat{M})
$$

This equivalent TMG system depends on the initial marking $M$ and the minimal T-semiflow $\boldsymbol{x}$ of the TWMG. Since it is necessary for us to use this transformation method, we present it in Algorithm 1. All notations in the algorithm are from previous definitions and $x_{o u t\left(p_{i}\right)}$ in Eq. (6) (resp. $x_{i n\left(p_{i}\right)}$ in Eq. (8)) represents the elementary T-semiflow component corresponding to transition $t_{\text {out }\left(p_{i}\right)}$ (resp. $\left.t_{i n\left(p_{i}\right)}\right)$.

Note that Eqs. (6) and (8) admit only one solution $\left(a_{s}, b_{s}\right.$ and $\left.c_{s}, d_{s}\right)$ for each value of $s$.

The structure of the equivalent TMG (i.e., the arcs connecting places and transitions) depends on the marking $M$ of the TWMG. However, this dependence is periodic as shown in the following proposition.

Proposition 2: Consider a TWMG $N$ with minimal T-semiflow $\boldsymbol{x}=\left(x_{1}, x_{2}, \ldots, x_{m}\right)^{T}$ and two possible initial markings $\boldsymbol{M}_{1}$ and $\boldsymbol{M}_{2}$. Let $\left\langle\hat{N}_{1}, \hat{\boldsymbol{M}}_{1}\right\rangle$ (resp., $\left\langle\hat{N}_{2}, \hat{\boldsymbol{M}}_{2}\right\rangle$ ) be the equivalent TMG obtained by Algorithm 1 with input $\left\langle N, \boldsymbol{M}_{1}\right\rangle$ (resp., $\left\langle N, \boldsymbol{M}_{2}\right\rangle$ ).

If for a place $p_{i} \in P$

$$
M_{2}\left(p_{i}\right)=M_{1}\left(p_{i}\right)+\xi \cdot v\left(p_{i}\right) \cdot x_{\text {out }\left(p_{i}\right)} \quad \text { with } \xi \in \mathbb{N},
$$

then the structure corresponding to $p_{i}$ in $\hat{N}_{1}$ and $\hat{N}_{2}$ is the same and the markings of the transformed places $p_{i}^{s}$ corresponding to $p_{i}$ in Eqs. (7) and (9) satisfy

$$
\hat{M}_{2}\left(p_{i}^{s}\right)=\hat{M}_{1}\left(p_{i}^{s}\right)+\xi
$$

Proof. Since $M_{1}\left(p_{i}\right)+x_{i n\left(p_{i}\right)} \cdot w\left(p_{i}\right)-x_{\text {out }\left(p_{i}\right)} \cdot v\left(p_{i}\right)=M_{1}\left(p_{i}\right)$, we have $x_{i n\left(p_{i}\right)} \cdot w\left(p_{i}\right)=x_{\text {out }\left(p_{i}\right)} \cdot v\left(p_{i}\right)$. If $w\left(p_{i}\right)>v\left(p_{i}\right), x_{i n\left(p_{i}\right)}<x_{\text {out }\left(p_{i}\right)}$ and $s=1, \ldots, x_{i n\left(p_{i}\right)}$, for marking $M_{1}\left(p_{i}\right)$ of place $p_{i}$, it holds that:

$$
\left\{\begin{array}{l}
a_{s} \cdot x_{\text {out }\left(p_{i}\right)}+b_{s}=\left\lfloor\frac{M_{1}\left(p_{i}\right)+w\left(p_{i}\right) \cdot(s-1)}{v\left(p_{i}\right)}\right\rfloor+1, \\
b_{s} \in\left\{1, \ldots, x_{\text {out }\left(p_{i}\right)}\right\}, a_{s} \in \mathbb{N},
\end{array}\right.
$$

${ }^{2}$ In the following, we will denote by $\chi(\boldsymbol{M})$ the cycle time of a TWMG system $\langle N, \boldsymbol{M}\rangle$ and by $\hat{\chi}(\hat{\boldsymbol{M}})$ the cycle time of the equivalent TMG system $\langle\hat{N}, \hat{\boldsymbol{M}}\rangle$. 


\begin{tabular}{l}
\hline Algorithm 1: Transformation from a TWMG to a TMG \\
Input: A TWMG system $\langle N, \boldsymbol{M}\rangle$.
\end{tabular}

Output: An equivalent TMG system $\langle\hat{N}, \hat{\boldsymbol{M}}\rangle$ such that $\chi(\boldsymbol{M})=\hat{\chi}(\hat{\boldsymbol{M}})$.

1: Compute the minimal T-semiflow $\boldsymbol{x}=\left(x_{1}, \ldots, x_{m}\right)^{T}$ of net $N$.

2: (Transformation of transitions). Replace each transition $t_{i} \in T$ by $x_{i}$ transitions, $t_{i}^{1}, t_{i}^{2}, \ldots, t_{i}^{x_{i}}$, with the same firing delay of $t_{i}$. These transitions are connected by an elementary circuit with all weights equal to 1 . Add $x_{i}$ places $q_{i}^{1}, q_{i}^{2}, \ldots, q_{i}^{x_{i}}$, where $q_{i}^{a}, a=1, \ldots, x_{i}-1$, is a place connecting transition $t_{i}^{a}$ to transition $t_{i}^{a+1}$ and $q_{i}^{x_{i}}$ is a place connecting transition $t_{i}^{x_{i}}$ to $t_{i}^{1}$. Only place $q_{i}^{x_{i}}$ contains one token and the other places are empty, i.e.,

$$
\left\{\begin{array}{l}
\hat{M}\left(q_{i}^{a}\right)=0, \forall i=1, \ldots, m, \forall a=1, \ldots, x_{i}-1, \\
\hat{M}\left(q_{i}^{x_{i}}\right)=1, \forall i=1, \ldots, m,
\end{array}\right.
$$

Thus there exist $m$ mono-marked circuits that are called intra transition sequential systems. They do not depend on the initial marking.

3: (Transformation of places: case 1). Replace each place $p_{i} \in P$ such that $w\left(p_{i}\right)>v\left(p_{i}\right)$ by $n_{i}=x_{i n\left(p_{i}\right)}$ places $p_{i}^{s}$, where for $s=1, \ldots, n_{i}$ :

$$
\left\{\begin{array}{l}
a_{s} \cdot x_{\text {out }\left(p_{i}\right)}+b_{s}=\left\lfloor\frac{M\left(p_{i}\right)+w\left(p_{i}\right) \cdot(s-1)}{v\left(p_{i}\right)}\right\rfloor+1, \\
b_{s} \in\left\{1, \ldots, x_{\text {out }\left(p_{i}\right)}\right\} \\
a_{s} \in \mathbb{N}
\end{array}\right.
$$

Place $p_{i}^{s}$ connects transition $t_{i n\left(p_{i}\right)}^{s}$ to transition $t_{\text {out }\left(p_{i}\right)}^{b_{s}}$ and contains $a_{s}$ tokens, i.e.,

$$
\hat{M}\left(p_{i}^{s}\right)=a_{s} .
$$

4: (Transformation of places: case 2). Replace each place $p_{i} \in P$ such that $w\left(p_{i}\right) \leq v\left(p_{i}\right)$ by $n_{i}=x_{\text {out }\left(p_{i}\right)}$ places $p_{i}^{s}$, where for $s=1, \ldots, n_{i}$ :

$$
\left\{\begin{array}{l}
c_{s} \cdot x_{i n\left(p_{i}\right)}+d_{s}=\left\lceil\frac{s \cdot v\left(p_{i}\right)-M\left(p_{i}\right)}{w\left(p_{i}\right)}\right\rceil, \\
d_{s} \in\left\{1, \ldots, x_{i n\left(p_{i}\right)}\right\} \\
c_{s} \in \mathbb{Z}_{\leq 0}
\end{array}\right.
$$

Place $p_{i}^{s}$ connects transition $t_{i n\left(p_{i}\right)}^{d_{s}}$ to transition $t_{o u t\left(p_{i}\right)}^{s}$ and contains $-c_{s}$ tokens, i.e.,

$$
\hat{M}\left(p_{i}^{s}\right)=-c_{s}
$$


and for marking $M_{2}\left(p_{i}\right)$ of place $p_{i}$,

$$
\begin{aligned}
& \left\{\begin{array}{l}
a_{s}^{\prime} \cdot x_{\text {out }\left(p_{i}\right)}+b_{s}^{\prime}= \\
\left\lfloor\frac{M_{1}\left(p_{i}\right)+\xi \cdot x_{\text {out }\left(p_{i}\right) \cdot v\left(p_{i}\right)+w\left(p_{i}\right) \cdot(s-1)}}{v\left(p_{i}\right)}\right\rfloor+1, \\
b_{s}^{\prime} \in\left\{1, \ldots, x_{\text {out }\left(p_{i}\right)}\right\}, \\
a_{s}^{\prime} \in \mathbb{N},
\end{array}\right. \\
& a_{s}^{\prime} \cdot x_{\text {out }\left(p_{i}\right)}+b_{s}^{\prime}=\left\lfloor\frac{M_{1}\left(p_{i}\right)+w\left(p_{i}\right) \cdot(s-1)}{v\left(p_{i}\right)}\right\rfloor+\xi \cdot x_{\text {out }\left(p_{i}\right)}+1,
\end{aligned}
$$

then

$$
a_{s}^{\prime} \cdot x_{\text {out }\left(p_{i}\right)}+b_{s}^{\prime}=\left(a_{s}+\xi\right) \cdot x_{\text {out }\left(p_{i}\right)}+b_{s}
$$

and

$$
\left\{\begin{array}{l}
a_{s}^{\prime}=a_{s}+\xi, \\
b_{s}^{\prime}=b_{s},
\end{array}\right.
$$

If $w\left(p_{i}\right) \leq v\left(p_{i}\right), x_{i n\left(p_{i}\right)} \geq x_{\text {out }\left(p_{i}\right)}$ and $k=1, \ldots, x_{\text {out }\left(p_{i}\right)}$, we can obtain the following equation

$$
\left\{\begin{array}{l}
c_{s}^{\prime}=c_{s}-\xi, \\
d_{s}^{\prime}=d_{s},
\end{array}\right.
$$

where $a_{s}$ and $-c_{s}$ represent the number of tokens in equivalent places and $b_{s}$ and $d_{s}$ represent the structure (input arc or output arc) of equivalent places. According to Eqs. (11) and (12), it follows that the equivalent structures of $M_{1}\left(p_{i}\right)$ and $M_{2}\left(p_{i}\right)$ are identical while

$$
\hat{M}_{2}\left(p_{i}^{s}\right)=\hat{M}_{1}\left(p_{i}^{s}\right)+\xi
$$

The previous result implies that the structure corresponding to place $p_{i}$ in the equivalent TMG is periodic with regard to $M\left(p_{i}\right)$ and the period $\phi_{i}$ is equal to $v\left(p_{i}\right) \cdot x_{\text {out }\left(p_{i}\right)}$ (or equivalently $w\left(p_{i}\right) \cdot x_{i n\left(p_{i}\right)}$ ).

The size of the equivalent TMG is ${ }^{3} O\left(|\boldsymbol{x}|_{1}\right)$. More precisely the number of transitions is $\hat{m}=|\boldsymbol{x}|_{1}$ and that of places is $\hat{n}=\sum_{i=1}^{n} n_{i}+|\boldsymbol{x}|_{1}$ which is less than or equal to $2|\boldsymbol{x}|_{1}$. Theoretically $|\boldsymbol{x}|_{1}$ can grow exponentially with respect to the net size. However, one finds that in practical examples, this is a quite reasonable number.

Example 1. We give an example in Fig. 2 and explain the process of transformation by assuming that the initial marking is $\boldsymbol{M}_{0}=(0,0,4)^{T}$.

Transformation of transitions: The minimal T-semiflow in Fig. 2 is $\boldsymbol{x}=(1,2,1)^{T}$. Then the transitions $t_{1}, t_{2}$ and $t_{3}$ are replaced by one transition, two transitions and one transition, respectively. Moreover, places $q$ 's to connect these transitions are added. The nets drawn by dotted lines in Fig. 3 correspond to the intra transition sequential systems.

\footnotetext{
${ }^{3}$ Here $|\boldsymbol{x}|_{1}$ denotes the 1-norm of T-semiflow $\boldsymbol{x}$.
} 
Transformation of places: Since place $p_{1}$ satisfies the condition $w\left(p_{1}\right)>v\left(p_{1}\right)$, it is replaced by place $p_{1}^{1}$ according to Algorithm 1. We compute $a_{1}$ and $b_{1}$ to determine the marking and structure of place $p_{1}^{1}$. Places $p_{2}$ and $p_{3}$ satisfy the condition $w\left(p_{i}\right) \leq v\left(p_{i}\right)(i=2,3)$, and then places $p_{2}$ and $p_{3}$ are replaced by $p_{2}^{1}$ and $p_{3}^{1}$, respectively. Markings and structures of $p_{2}^{1}$ and $p_{3}^{1}$ are computed by Eq. (8).

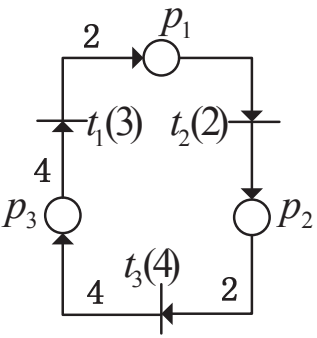

Fig. 2. TWMG of Example 1.

Fig. 3 shows the equivalent TMG with the initial marking $\boldsymbol{M}=(0,0,4)^{T}$. There are totally four transitions and seven places. From Proposition 2, we can compute the period of each place $\phi_{1}=2, \phi_{2}=2$ and $\phi_{3}=4$. For the marking $\boldsymbol{M}^{\prime}=\left(2 \xi_{1}, 2 \xi_{2}, 4 \xi_{3}\right)^{T}$, one can easily check that the structure of the equivalent TMG is identical to that of the net shown in Fig. 3 while the markings of equivalent places are $\hat{M}^{\prime}\left(p_{1}^{1}\right)=\xi_{1}, \hat{M}^{\prime}\left(p_{2}^{1}\right)=\xi_{2}$ and $\hat{M}^{\prime}\left(p_{3}^{1}\right)=\xi_{3}$.

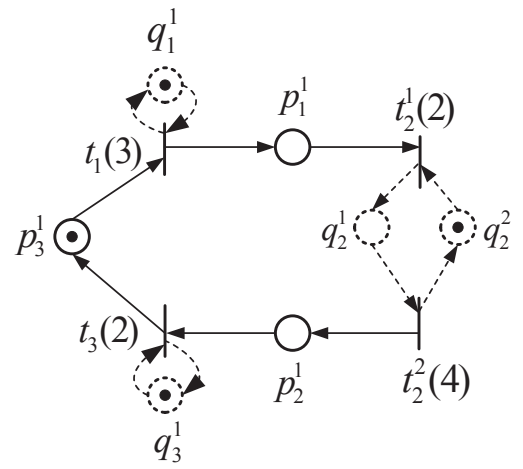

Fig. 3. TMG equivalent to the TWMG in Fig. 2 for initial marking $\boldsymbol{M}_{0}=(0,0,4)^{T}$.

\section{Problem Statement}

In this paper, the cycle time optimization problem of a TWMG is considered. We aim to find an initial marking $M$ such that the weighted sum of tokens in places is less than or equal to a given value. Among all feasible solutions, we look for those that minimize the cycle time, i.e., maximize the throughput. 
We consider a non-negative cost vector $\boldsymbol{y} \in \mathbb{N}^{|P|}$ that is a P-semiflow since the value of $\boldsymbol{y}^{T} \cdot \boldsymbol{M}$ at every reachable marking $\boldsymbol{M}^{\prime} \in R(N, \boldsymbol{M})$ is an invariant. In particular, if $\Gamma$ denotes the set of elementary circuits of the net, we can write the cost vector $\boldsymbol{y}$ as the weighted sum of all minimal P-semiflows, i.e.,

$$
\boldsymbol{y}=\sum_{\gamma \in \Gamma} \lambda_{\gamma} \cdot \boldsymbol{y}_{\gamma},
$$

where $\boldsymbol{y}_{\gamma}$ denotes the minimal P-semiflow of circuit $\gamma$ and $\lambda_{\gamma}$ represents the cost of the resources modeled by tokens in the support of $\boldsymbol{y}_{\gamma}$.

Problem 1: Let $N$ be a TWMG with a set of the elementary circuits $\Gamma$ and $y \in \mathbb{N}^{|P|}$ be a non-negative cost vector as defined in Eq. (13). Given a positive real number $R$ that represents the upper bound on the cost of available resources, we look for an initial marking $\boldsymbol{M}$ which minimizes the cycle time $\chi(\boldsymbol{M})$ :

$$
\begin{aligned}
& \min \chi(\boldsymbol{M}) \\
& \text { s.t. } \\
& \qquad \boldsymbol{y}^{T} \cdot \boldsymbol{M} \leq R .
\end{aligned}
$$

\section{CYCLE TIME OPTIMIZATION FOR TWMGs: A MIXED ILPP SOLUTION}

We propose here a mixed ILPP to solve the cycle time optimization problem for TWMGs. We first give some conditions under which the optimization problem admits a finite solution. Then, we show the general idea on which our approach is based. Some techniques are introduced in Subsection IV-C to reduce the equivalent TMGs structures. Finally, we formulate the proposed approach in Subsection IV-D.

\section{A. Existence of a finite solution}

Let us first recall some basic results regarding the liveness of TWMGs.

Proposition 3: (Teruel et al. [3]) A TWMG is live iff each elementary circuit is live.

For a TWMG, the complexity of checking liveness and determining the minimal number of tokens that ensures the liveness remains open. Some sufficient conditions ensuring liveness are proposed in [3], [41], and [42]. Teruel et al. [3] proposed a sufficient condition for the liveness of a weighted circuit $\gamma$. Let us preliminarily define the greatest dead marking of circuit $\gamma$ as $\boldsymbol{M}_{\gamma}^{D}=\left(v\left(p_{1}\right)-1, v\left(p_{2}\right)-1, \ldots, v\left(p_{n}\right)-1\right)^{T}$ (recall that $v\left(p_{i}\right)$ is the weight of the output arc of place $p_{i}$ ).

Proposition 4: (Teruel et al. [3]) Let $\gamma$ be a weighted circuit with greatest dead marking $\boldsymbol{M}_{\gamma}^{D}$ and $\boldsymbol{y}_{\gamma}$ be its minimal P-semiflow. The weighted circuit $\gamma$ is live at marking $M \in \mathbb{N}^{|P|}$, if it holds

$$
\boldsymbol{y}_{\gamma}^{T} \cdot \boldsymbol{M}>\boldsymbol{y}_{\gamma}^{T} \cdot \boldsymbol{M}_{\gamma}^{D}
$$

Based on the previous results, we now present a sufficient condition concerning the existence of a finite solution to the considered optimization problem. 
Proposition 5: Let $\boldsymbol{M}$ be the initial marking of a TWMG, $\boldsymbol{y}=\sum_{\gamma \in \Gamma} \lambda_{\gamma} \cdot \boldsymbol{y}_{\gamma}$ be a cost vector as defined in Eq. (13), $M_{\gamma}^{D}$ be the greatest dead marking of circuit $\gamma$, and $R$ be a positive real number that represents the upper bound on the cost of available resources. Problem 1 as defined in Eq. (14) has a finite solution if $R \geq R^{*}$, where $R^{*}$ is a positive real number such that:

$$
\begin{aligned}
R^{*}= & \min \quad \boldsymbol{y}^{T} \cdot \boldsymbol{M}, \\
\text { s.t. } & \\
& \boldsymbol{y}_{\gamma}^{T} \cdot \boldsymbol{M}>\boldsymbol{y}_{\gamma}^{T} \cdot \boldsymbol{M}_{\gamma}^{D} \quad(\forall \gamma \in \Gamma) .
\end{aligned}
$$

Proof. If for any $\gamma \in \Gamma, \boldsymbol{y}_{\gamma}^{T} \cdot \boldsymbol{M}>\boldsymbol{y}_{\gamma}^{T} \cdot \boldsymbol{M}_{D}$ holds, we conclude that each circuit of the TWMG is live according to Proposition 4. Then, the TWMG is necessarily live according to Proposition 3 and its cycle time will be finite.

Sauer [29] proved that the lower bound of the cycle time is

$$
\chi^{\prime}=\max \left\{x_{i} \cdot \delta_{i}, t_{i} \in T\right\}
$$

where $\boldsymbol{x}$ is the minimal T-semiflow.

\section{B. General idea}

Giua et al. [12] proved that for a TMG the solution ${ }^{4}$ of Problem 1 can be computed by solving the following mixed ILPP:

$$
\begin{aligned}
& \max \beta \\
& \text { s.t. } \\
& \left\{\begin{array}{l}
\boldsymbol{C} \cdot \boldsymbol{\alpha}-\boldsymbol{P r} \boldsymbol{e} \cdot \boldsymbol{\delta} \cdot \beta+\boldsymbol{M} \geq 0 \\
\boldsymbol{y}^{T} \cdot \boldsymbol{M} \leq R
\end{array}\right.
\end{aligned}
$$

with variables $M \in \mathbb{N}^{n}, \beta \in \mathbb{R}^{+}$and $\boldsymbol{\alpha} \in \mathbb{R}^{m}$. It provides the optimal solution $M$ and the corresponding maximal throughput $\beta$ (i.e., the inverse of cycle time $1 / \chi(\boldsymbol{M})$ ), and $\boldsymbol{\alpha}$ has no obvious physical meaning.

For TWMGs one way to find the optimal solution of Problem 1 is to enumerate all possible equivalent TMGs and solve a mixed ILPP (16) for each of them to find a marking which has the maximal throughput. However, there are two main problems.

- The number of TMG structures equivalent to a TWMG may be very large. This issue is addressed in Section IV-C.

- We have to add in Eq. (16) a series of constraints to ensure the marking $\hat{M}$ that we find for a given net structure $\hat{N}$ is consistent with the marking $M$ of the original TWMG. We discuss this issue in Section IV-D.

${ }^{4}$ The mixed ILPP in Eq. (16) provides a solution under infinite server semantics while here we consider single server. However, the equivalent TMGs constructed by Algorithm 1 are such that the enabling degree of transitions is at most equal to one: this means that their behavior is the same under both infinite and single server semantics. 


\section{Reduction of equivalent TMG structures}

According to Proposition 2, for each place $p_{i} \in P$ of a TWMG system $\langle N, \boldsymbol{M}\rangle$, the structure corresponding to place $p_{i}$ in the equivalent TMG is periodic with respect to $M\left(p_{i}\right)$ and the period is $\phi_{i}$. Thus, we should compute the equivalent structures for initial marking $M\left(p_{i}\right)=0,1, \ldots, \phi_{i}-1$.

We note that the set of possible markings of place $p_{i}$ can be partitioned into $\phi_{i}$ subsets such that

$$
\bigcup_{k_{i}=0}^{\phi_{i}-1} \mathcal{M}_{p_{i}}^{k_{i}}=\mathbb{N} \text {, where }\left\{k_{i}+\xi \cdot \phi_{i} \mid \xi \in \mathbb{N}\right\} \quad=\mathcal{M}_{p_{i}}^{k_{i}},
$$

and all makings of $p_{i}$ in the same partition $\mathcal{M}_{p_{i}}^{k_{i}}$ correspond to the same equivalent structure.

For each place $p_{i} \in P$, we define $\mathcal{N}_{i}=\left\{0, \ldots, \phi_{i}-1\right\}$. Then the set of markings of a TWMG can be partitioned into several subsets

$$
\bigcup_{\left(k_{1}, \ldots, k_{n}\right) \in \mathcal{N}_{1} \times \cdots \times \mathcal{N}_{n}} \mathcal{M}_{p_{1}}^{k_{1}} \times \mathcal{M}_{p_{2}}^{k_{2}} \times \ldots \times \mathcal{M}_{p_{n}}^{k_{n}}=\mathbb{N}^{n}
$$

For each vector $\boldsymbol{k}=\left(k_{1}, \ldots, k_{n}\right) \in \mathcal{N}_{1} \times \cdots \times \mathcal{N}_{n}$ corresponding to partition $\mathcal{M}_{p_{1}}^{k_{1}} \times \mathcal{M}_{p_{2}}^{k_{2}} \times \ldots \times \mathcal{M}_{p_{n}}^{k_{n}}$, the equivalent TMGs for all markings in this partition are the same. The total number of such structures (i.e., partitions) is

$$
\Phi=\prod_{p_{i} \in P} \phi_{i}
$$

Note that the number of equivalent structures given by Eq. (19) is usually large. We look for more efficient solutions that only require to consider a subset of these structures (i.e., partitions). To reach this goal, the following result is useful.

Lemma 1: (Marchetti and Munier [41]) For a WMG, the initial marking $M\left(p_{i}\right)$ of any place $p_{i}$ can be replaced by $M^{\star}\left(p_{i}\right)=\left\lfloor\frac{M\left(p_{i}\right)}{g c d_{p_{i}}}\right\rfloor \cdot g c d_{p_{i}}$ tokens without any influence on the precedence constraints induced by $p_{i}$. $\diamond$

In fact, if $M\left(p_{i}\right)$ is not a multiple of $\operatorname{gcd}_{p_{i}}$, there will always be $M\left(p_{i}\right)-M^{\star}\left(p_{i}\right)$ tokens remaining in place $p_{i}$ that will never be used in the firing of the output transition of place $p_{i}$. As a result, we can deduce that the cycle time at $\boldsymbol{M}_{0}$ and $\boldsymbol{M}_{0}^{\star}$ are the same.

Let us see the example in Fig. 2. We assume the initial marking $\boldsymbol{M}_{0}=(0,0,11)^{T}$.

$$
M_{0}^{\star}\left(p_{3}\right)=\left\lfloor\frac{M_{0}\left(p_{3}\right)}{g c d_{p_{3}}}\right\rfloor \cdot g c d_{p_{3}}=\left\lfloor\frac{11}{4}\right\rfloor \cdot 4=8
$$

Then $\boldsymbol{M}_{0}^{\star}=(0,0,8)^{T}$ and we can check that the equivalent TMGs of $\boldsymbol{M}_{0}$ and $\boldsymbol{M}_{0}^{\star}$ are the same, which implies that the cycle times of system $\left\langle N, \boldsymbol{M}_{0}\right\rangle$ and system $\left\langle N, \boldsymbol{M}_{0}^{\star}\right\rangle$ are identical, i.e., $\chi\left(\boldsymbol{M}_{0}\right)=\chi\left(\boldsymbol{M}_{0}^{\star}\right)$.

From Lemma 1, when looking for an optimal solution for Problem 1, we may restrict our analysis to the markings that belong to a restricted number of partitions where the token content of each place $p_{i}$ is a multiple of $\operatorname{gcd}_{p_{i}}$. Hence the number of meaningful subsets in Eq. (17) can be reduced as follows:

$$
\begin{gathered}
\bigcup_{k_{i}=0}^{\frac{\phi_{i}}{g c d_{p_{i}}}-1} \overline{\mathcal{M}}_{p_{i}}^{k_{i}} \subseteq \mathbb{N}, \\
\overline{\mathcal{M}}_{p_{i}}^{k_{i}}=\left\{k_{i} \cdot g c d_{p_{i}}+\xi \cdot \phi_{i} \mid \xi \in \mathbb{N}\right\} .
\end{gathered}
$$


We define $\overline{\mathcal{N}}_{i}=\left\{0, \ldots, \frac{\phi_{i}}{g c d_{p_{i}}}-1\right\}$ and the set of markings of a TWMG in Eq. (18) can be redefined as

$$
\mathcal{M}_{\text {opt }}=\bigcup_{\left(k_{1}, \ldots, k_{n}\right) \in \mathcal{N}_{1}^{\prime} \times \cdots \times \mathcal{N}_{n}^{\prime}} \overline{\mathcal{M}}_{p_{1}}^{k_{1}} \times \overline{\mathcal{M}}_{p_{2}}^{k_{2}} \times \ldots \times \overline{\mathcal{M}}_{p_{n}}^{k_{n}} \subseteq \mathbb{N}^{n}
$$

where the number of partitions is reduced to

$$
\Phi^{\prime}=\prod_{p_{i} \in P} \frac{\phi_{i}}{g c d_{p_{i}}} .
$$

In the following, for the sake of simplicity, we rename the partitions defined in Eq. (21) and write

$$
\mathcal{M}_{\text {opt }}=\bigcup_{j=1}^{\Phi^{\prime}} \mathcal{M}_{j}
$$

where

$$
\mathcal{M}_{j}=\overline{\mathcal{M}}_{p_{1}}^{k_{j, 1}} \times \overline{\mathcal{M}}_{p_{2}}^{k_{j, 2}} \times \ldots \times \overline{\mathcal{M}}_{p_{n}}^{k_{j, n}}
$$

i.e., partition $j$ is characterized by the $n$-tuple $\left(k_{j, 1}, \ldots, k_{j, n}\right)$.

Let us see the example in Fig. 2. We have $\operatorname{gcd}_{p_{1}}=1, \operatorname{gcd}_{p_{2}}=1, \operatorname{gcd}_{p_{3}}=4, \phi_{1}=2, \phi_{2}=2$, and $\phi_{3}=4$. The number of partitions is $\Phi=16$, while the number of meaningful partitions is $\Phi^{\prime}=4$, which is significantly smaller.

\section{The mixed ILPP solution for TWMGs}

We now show how it is possible to solve Problem 1 by assuming that the unknown initial marking $M$ of the TWMG belongs to a generic partition $\mathcal{M}_{j}$ shown in Eq. (24).

In this case, due to the special equivalent structure of a marking $M \in \mathcal{M}_{j}$ in Eq. (20), Problem 1 can be rewritten as

$$
\begin{aligned}
& \min \chi(\boldsymbol{M}) \\
& \text { s.t. } \\
& \left\{\begin{array}{l}
\boldsymbol{y}^{T} \cdot \boldsymbol{M} \leq R, \\
M\left(p_{i}\right)=k_{j, i} \cdot g c d_{p_{i}}+\xi_{j, i} \cdot \phi_{i}, \forall p_{i} \in P, \\
\xi_{j, i} \in \mathbb{N},
\end{array}\right.
\end{aligned}
$$

We define the vector $\boldsymbol{\xi}_{j}=\left(\xi_{j, 1}, \ldots, \xi_{j, n}\right)^{T}$ and for each place $p_{i}$ with an initial marking

$$
M\left(p_{i}\right)=k_{j, i} \cdot g_{c d}, k_{p_{i}, i}=0, \ldots, \frac{\phi_{i}}{g c d_{p_{i}}}-1,
$$

we compute

- the equivalent structure of place $p_{i}$, i.e., places $p_{i}^{1}, \ldots, p_{i}^{n_{i}}$,

- the initial markings correspond to Eq. (25), i.e., $\hat{M}\left(p_{i}^{1}\right)=\mu_{j}\left(p_{i}^{1}\right), \ldots, \hat{M}\left(p_{i}^{n_{i}}\right)=\mu_{j}\left(p_{i}^{n_{i}}\right)$.

Thus for each partition $\mathcal{M}_{j}$ given in Eq. (24), we can compute the equivalent net structure $\hat{N}_{j}$, incidence matrix $\hat{\boldsymbol{C}}_{j}$ and pre-incidence $\boldsymbol{P} \hat{\boldsymbol{r}} \boldsymbol{e}_{j}$. 
Proposition 6: For each partition $\mathcal{M}_{j}$ in Eq. (24), we consider the following mixed ILPP

$$
\begin{aligned}
& \max \beta_{j} \\
& \text { s.t. } \\
& \begin{cases}\hat{\boldsymbol{C}}_{j} \cdot \hat{\boldsymbol{\alpha}}_{j}-\hat{\boldsymbol{P}} \boldsymbol{r}_{j} \cdot \hat{\boldsymbol{\delta}}_{j} \cdot \beta_{j}+\hat{\boldsymbol{M}}_{j} \geq 0, & (a) \\
\boldsymbol{y}^{T} \cdot \boldsymbol{M}_{j} \leq R, & (b) \\
M_{j}\left(p_{i}\right)=k_{j, i} \cdot g c d_{p_{i}}+\xi_{j, i} \cdot \phi_{i}, \forall p_{i} \in P, & (c) \\
\hat{M}_{j}\left(p_{i}^{s}\right)=\mu_{j}\left(p_{i}^{s}\right)+\xi_{j, i}, s=1, \ldots, n_{i}, & (d) \\
\hat{M}_{j}\left(q_{i}^{a}\right)=0, \forall i=1, \ldots, m, \forall a=1, \ldots, x_{i}-1,(e) \\
\hat{M}_{j}\left(q_{i}^{x_{i}}\right)=1, \forall i=1, \ldots, m, \\
\xi_{j, i} \in \mathbb{N},\end{cases}
\end{aligned}
$$

with variables ${ }^{5} \beta_{j} \in \mathbb{R}_{\geq 0}, \boldsymbol{M}_{j} \in \mathbb{N}^{n}, \hat{\boldsymbol{M}}_{j} \in \mathbb{N}^{\hat{n}}, \hat{\boldsymbol{\alpha}}_{j} \in \mathbb{R}^{\hat{m}}$, and $\boldsymbol{\xi}_{j} \in \mathbb{N}^{n}$. Let $\left(\beta_{j}^{*}, \boldsymbol{M}_{j}^{*}, \hat{\boldsymbol{M}}_{j}^{*}, \hat{\boldsymbol{\alpha}}_{j}^{*}, \boldsymbol{\xi}_{j}^{*}\right)$ be an optimal solution of Eq. (26). Thus $\boldsymbol{M}_{j}^{*}$ is also an optimal solution of Problem 1 restricted to partition $\mathcal{M}_{j}$.

Proof. The constraint (a) adopted from Eq. (16) can provide an optimal solution if $\hat{\boldsymbol{C}_{j}}, \boldsymbol{P} \hat{\boldsymbol{r}} \boldsymbol{e}_{\boldsymbol{j}}$ and $\hat{\boldsymbol{\delta}_{j}}$ are given. The constraint $(b)$ specifies that the weighted sum of tokens in places cannot exceed the upper bound of the number of available resources, and the constraint $(c)$ specifies that feasible markings should be restricted to partition $\mathcal{M}_{j}$.

As shown in Eqs. (5) and (10), the marking $\hat{M}_{j}$ of the equivalent TMG should be consistent with the marking $\boldsymbol{M}_{j}$ of the TWMG; this is ensured by constraints $(d),(e)$ and $(f)$.

In [12] the authors proved that the mixed ILPP can obtain an optimal solution for the cycle time optimization problem. Thus, $\left(\beta_{j}^{*}, \boldsymbol{M}_{j}^{*}, \hat{\boldsymbol{M}}_{j}^{*}, \hat{\boldsymbol{\alpha}}_{j}^{*}, \boldsymbol{\xi}_{j}^{*}\right)$ is an optimal solution of Problem 1 resticted to partition $\mathcal{M}_{j}$.

Note that the mixed ILPP in Eq. (26) has $|\boldsymbol{x}|_{1}+n+1$ variables and at most $6|\boldsymbol{x}|_{1}+n+1$ constrains, where $n$ denotes the number of places of a TWMG.

Property 1: Any marking $\boldsymbol{M}$ that produces a cycle time $\chi(\boldsymbol{M})=\chi^{\prime}$ as defined in Eq. (15) and satisfies $\boldsymbol{y}^{T} \cdot \boldsymbol{M} \leq R$ is an optimal solution.

Proof. According to Eq. (15), once we obtain a marking $\boldsymbol{M}$ which has the cycle time $\chi(\boldsymbol{M})=\chi^{\prime}$, no more reduction can be obtained no matter how many resources we increase. Obviously, the throughput is maximal and $M$ is an optimal initial marking.

We can summarize the proposed procedure in Algorithm 2.

Proposition 7: The output of Algorithm 2 provides an optimal solution of Problem 1.

Proof. It is obvious that if we solve Eq. (26) for each partition, among all the optimal solutions, we can obtain the maximal throughput

$$
\beta=\max _{j=1, \ldots, \Phi^{\prime}} \beta_{j}^{*}
$$

and the corresponding marking $\boldsymbol{M}$. The global optimal solutions of Problem 1 are $\boldsymbol{M}$ and $\chi(\boldsymbol{M})=1 / \beta$.

${ }^{5}$ Recall that $\hat{n}$ (resp. $\hat{m}$ ) is the number of places (resp. transitions) of the equivalent TMG. 


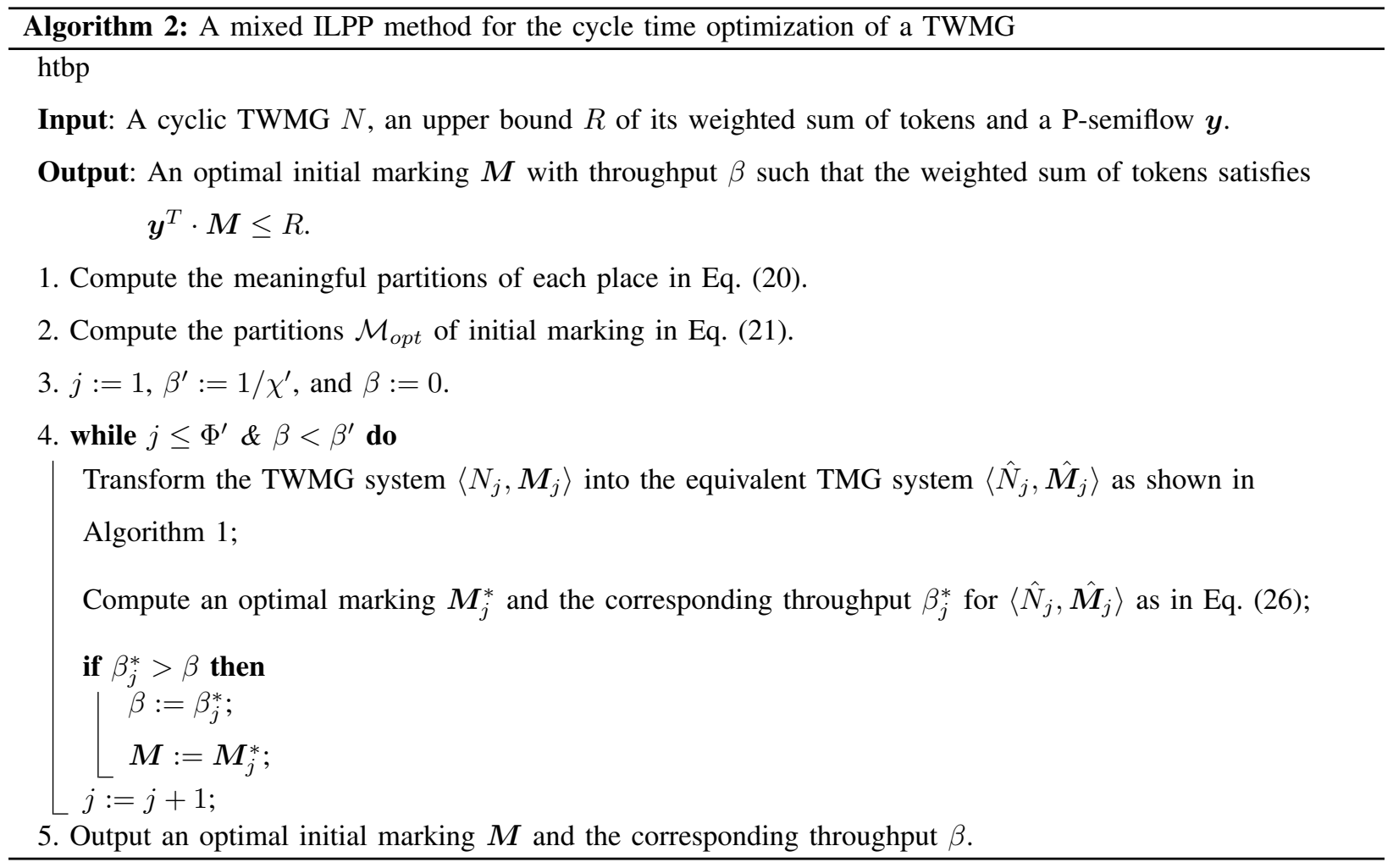

The mechanism of Algorithm 2 can be explained by Fig. 4. From a theoretical point of view, we should compute

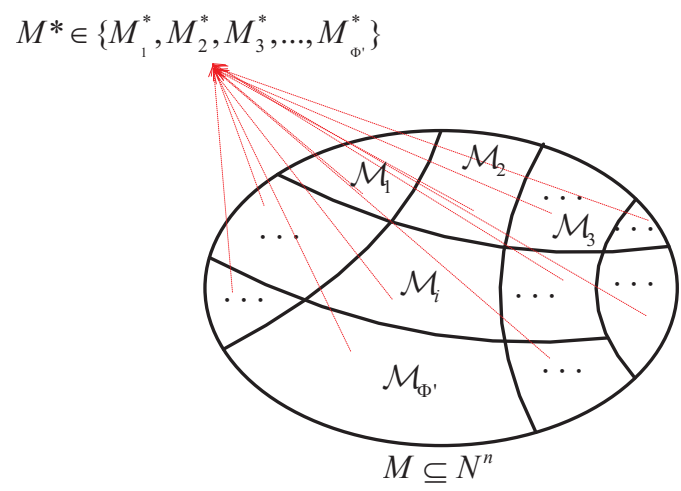

Fig. 4. Mechanism of Algorithm 2.

the solutions for all $\Phi^{\prime}$ partitions. However, in practical if we find a marking $M$ whose cycle time converges to the lower bound, there is no need to do more computations. According to Property 1, we can conclude that marking $M$ is an optimal solution.

Example 2. Let us consider the TWMG in Fig. 5. The minimal T-semiflows is $\boldsymbol{x}=(1,3,1,1)^{T}$, while the minimal P-semiflows are $\boldsymbol{y}_{1}=(1,0,6,1,0)^{T}$ and $\boldsymbol{y}_{2}=(0,2,2,0,1)^{T}$. Therefore, we choose the P-semiflow $\boldsymbol{y}=$ 
$\boldsymbol{y}_{1}+\boldsymbol{y}_{2}=(1,2,8,1,1)^{T}$. We have $\phi_{1}=6, \phi_{2}=1, \phi_{3}=1, \phi_{4}=6, \phi_{5}=2, \operatorname{gcd}_{p_{1}}=2, \operatorname{gcd}_{p_{2}}=1, \operatorname{gcd}_{p_{3}}=1$, $g c d_{p_{4}}=2$, and $g c d_{p_{5}}=2$. The number of variables is equal to 12 and the number of constrains is equal to 28 . The markings of the TWMG are partitioned into $\Phi^{\prime}=9$ subsets.

$$
\left\{\begin{array}{l}
\mathcal{M}_{1}=\left(6 \xi_{1,1}, \xi_{1,2}, \xi_{1,3}, 6 \xi_{1,4}, 2 \xi_{1,5}\right)^{T} \\
\mathcal{M}_{2}=\left(2+6 \xi_{2,1}, \xi_{2,2}, \xi_{2,3}, 6 \xi_{2,4}, 2 \xi_{2,5}\right)^{T} \\
\mathcal{M}_{3}=\left(4+6 \xi_{3,1}, \xi_{3,2}, \xi_{3,3}, 6 \xi_{3,4}, 2 \xi_{3,5}\right)^{T} \\
\mathcal{M}_{4}=\left(6 \xi_{4,1}, \xi_{4,2}, \xi_{4,3}, 2+6 \xi_{4,4}, 2 \xi_{4,5}\right)^{T} \\
\mathcal{M}_{5}=\left(6 \xi_{5,1}, \xi_{5,2}, \xi_{5,3}, 4+6 \xi_{5,4}, 2 \xi_{5,5}\right)^{T} \\
\mathcal{M}_{6}=\left(2+6 \xi_{6,1}, \xi_{6,2}, \xi_{6,3}, 2+6 \xi_{6,4}, 2 \xi_{6,5}\right)^{T} \\
\mathcal{M}_{7}=\left(2+6 \xi_{7,1}, \xi_{7,2}, \xi_{7,3}, 4+6 \xi_{7,4}, 2 \xi_{7,5}\right)^{T} \\
\mathcal{M}_{8}=\left(4+6 \xi_{8,1}, \xi_{8,2}, \xi_{8,3}, 2+6 \xi_{8,4}, 2 \xi_{8,5}\right)^{T} \\
\mathcal{M}_{9}=\left(4+6 \xi_{9,1}, \xi_{9,2}, \xi_{9,3}, 4+6 \xi_{9,4}, 2 \xi_{9,5}\right)^{T}
\end{array}\right.
$$

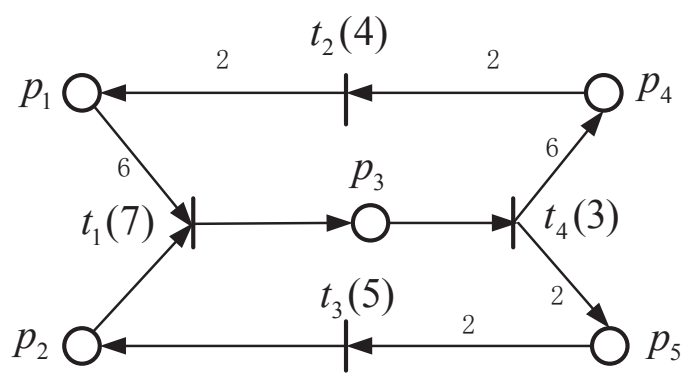

Fig. 5. TWMG of Example 2.

Let $R=20$ be the available cost of resources and problems of the form (26) can be immediately formulated for each partition $\mathcal{M}_{i}(i=1, \ldots, 9)$. In the following equation, we will show the mixed ILPP for partition $\mathcal{M}_{1}$. 


$$
\begin{aligned}
& \max \beta_{1} \\
& \text { s.t. } \\
& \left\{\begin{array}{l}
\hat{\boldsymbol{C}}_{1} \cdot \hat{\boldsymbol{\alpha}}_{1}-\hat{\boldsymbol{P \boldsymbol { r }}} \boldsymbol{e}_{1} \cdot \hat{\boldsymbol{\delta}}_{1} \cdot \beta_{1}+\hat{\boldsymbol{M}}_{1} \geq 0, \\
\boldsymbol{y}^{T} \cdot \boldsymbol{M}_{1} \leq 20, \\
M_{1}\left(p_{1}\right)=6 \xi_{1,1}, M_{1}\left(p_{2}\right)=\xi_{1,2}, M_{1}\left(p_{3}\right)=\xi_{1,3}, \\
M_{1}\left(p_{4}\right)=6 \xi_{1,4}, M_{1}\left(p_{5}\right)=2 \xi_{1,5}, \\
\hat{M}_{1}\left(p_{1}^{1}\right)=\xi_{1,1}, \hat{M}_{1}\left(p_{2}^{1}\right)=\xi_{1,2}, \hat{M}_{1}\left(p_{3}^{1}\right)=\xi_{1,3}, \\
\hat{M}_{1}\left(p_{4}^{1}\right)=\xi_{1,4}, \hat{M}_{1}\left(p_{5}^{1}\right)=\xi_{1,5}, \\
\hat{M}_{1}\left(q_{2}^{1}\right)=0, \hat{M}_{1}\left(q_{2}^{2}\right)=0, \\
\hat{M}_{1}\left(q_{1}^{1}\right)=1, \hat{M}_{1}\left(q_{2}^{3}\right)=1, \hat{M}_{1}\left(q_{3}^{1}\right)=1, \hat{M}_{1}\left(q_{4}^{1}\right)=1, \\
\xi_{1, i} \in \mathbb{N}, i=1, \ldots, 5 .
\end{array}\right.
\end{aligned}
$$

TABLE I

OPTIMAL SOLUTION OF EXAMPLE 2.

\begin{tabular}{cccccc}
\hline $\boldsymbol{M}_{1}$ & $\beta$ & $\chi\left(\boldsymbol{M}_{1}\right)$ & $\chi^{\prime}$ & $\boldsymbol{y}^{T} \cdot \boldsymbol{M}_{1}$ & $R$ \\
\hline$(6,1,1,0,0)^{T}$ & 0.083 & 12 & 12 & 16 & 20 \\
\hline
\end{tabular}

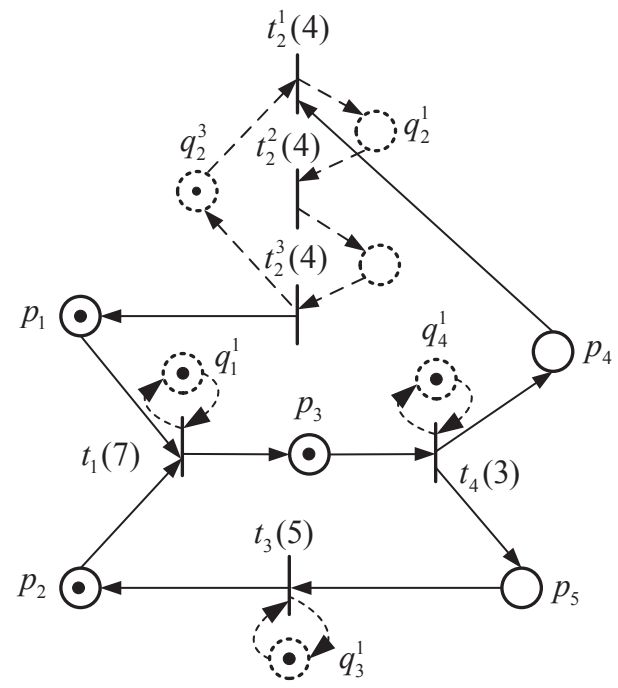

Fig. 6. Equivalent TMG for initial marking $\boldsymbol{M}=(6,1,1,0,0)^{T}$.

The solutions of Eq. (27) are $\beta_{1}=0.083$ and $\boldsymbol{M}_{1}=(6,1,1,0,0)^{T}$ and the equivalent TMG is depicted in Fig. 
6. We can observe from Table I that the cycle time $\chi\left(\boldsymbol{M}_{1}\right)$ is equal to the lower bound $\chi^{\prime}$. According to Property 1 , this solution is also globally optimal.

\section{EXTENSION OF THE BASIC APPROACH}

The aim of this section is to further improve the basic approach presented in Section IV by reducing the computation complexity of Algorithm 2 and by considering a slightly more general cycle time optimization problem. We first prove that the number of equivalent TMG structures to be analysed can be further reduced by exploring the net structure. Then, we discuss the more general optimization problem.

\section{A. Further reduction of equivalent TMG structures}

In this subsection, we will study the possibility to further reduce the number of equivalent TMG structures in Eq. (22). Firstly, we give an example in Fig. 7 to illustrate the main idea.

As one can see, the periods of places $p_{1}$ and $p_{2}$ are $\phi_{1}=6$ and $\phi_{2}=6$, respectively. Thus, the number of equivalent TMG structures is $\Phi^{\prime}=36$. However, if the number of tokens in place $p_{1}$ satisfies the condition $M\left(p_{1}\right) \geq 2$, we can always fire $t_{2}$ as many times as possible. Then, we can restrict our attention to partitions satisfying $M\left(p_{1}\right)<2$, i.e., $M\left(p_{1}\right)=0$ or $M\left(p_{1}\right)=1$. As a result, to find the optimal solution, we need only study 12 equivalent TMG structures rather than 36 . In the following, some propositions are given to reduce the partition as much as possible.

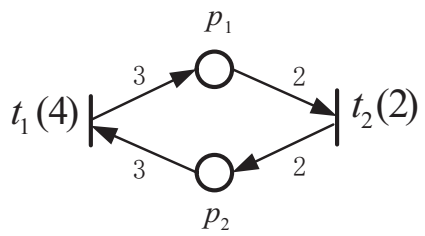

Fig. 7. An illustrative example to further reduce the equivalent structures.

Proposition 8: Let $N$ be a TWMG consisting of only one circuit and described by the following sequence: $p_{1} \stackrel{v\left(p_{1}\right)}{\longrightarrow} t_{1} \stackrel{w\left(p_{2}\right)}{\longrightarrow} p_{2} \stackrel{v\left(p_{2}\right)}{\longrightarrow} \cdots \stackrel{v\left(p_{n}\right)}{\longrightarrow} t_{n} \stackrel{w\left(p_{1}\right)}{\longrightarrow} p_{1}$. The number of partitions can be reduced to

$$
\Phi^{\prime \prime}=\frac{\phi_{n}}{g c d_{p_{n}}} \times \prod_{i=1}^{n-1} \frac{v\left(p_{i}\right)}{g c d_{p_{i}}} .
$$

Proof. For any live marking $\boldsymbol{M}$, we fire at $\boldsymbol{M}$ the transition $t_{1}$ as many times as we can. Next we fire $t_{2}$ as many times as we can, and so on, until we fire the transition $t_{n-1}$ leaving on the place $p_{n}$ the maximal number of tokens that can be put without firing $t_{n}$.

Then, we obtain a new marking $\boldsymbol{M}^{\prime}$ such that the number of tokens in each place is $M^{\prime}\left(p_{i}\right)<v\left(p_{i}\right)(i=$ $1, \ldots, n-1)$, i.e.,

$$
M^{\prime}\left(p_{i}\right) \in\left\{0, g c d_{p_{i}}, \ldots, v\left(p_{i}\right)-g c d_{p_{i}}\right\}, i=1, \ldots, n-1,
$$


and $M^{\prime}\left(p_{n}\right) \in \mathbb{N}$.

As a consequence, the number of equivalent TMG structures can be reduced to Eq. (28). By comparing Eq. (28) with Eq. (22), we can conclude that $\Phi^{\prime \prime} \leq \Phi^{\prime}$ is true due to $v\left(p_{i}\right) \leq \phi_{i}=v\left(p_{i}\right) \cdot x_{\text {out }}\left(p_{i}\right)$.

Now we will discuss how to reduce the partitions of a TWMG which consists of more than one single circuit. In the following, these notations are used.

- $T^{*}$ : the set of transitions of $N$ which have only one input place.

- $P^{*}$ : the set of pre-places of $T^{*}$.

Proposition 9: Let $N$ be a TWMG with $n$ places and $m$ transitions. The number of partitions of $N$ in Eq. (22) can be reduced to

$$
\Phi^{\prime \prime \prime}=\prod_{p_{j} \in P \backslash P^{*}} \frac{\phi_{j}}{g c d_{p_{j}}} \times \prod_{p_{i} \in P^{*}} \frac{v\left(p_{i}\right)}{g c d_{p_{i}}} .
$$

Proof. Let place $p_{i}$ belong to $P^{*}$ and transition $t_{\text {out }\left(p_{i}\right)}$ be the output transition of $p_{i}$. Thus, $p_{i}$ is the decisive place of transition $t$, i.e., the firing of $t_{\text {out }\left(p_{i}\right)}$ is only decided by $p_{i}$. We fire $t_{\text {out }\left(p_{i}\right)}$ as many times as possible and the final marking of place $p_{i}$ will satisfy the condition $M\left(p_{i}\right)<v\left(p_{i}\right)$. The number of meaningful subsets in Eq. (20) will be

$$
\begin{gathered}
\bigcup_{k_{i}=0}^{\frac{v\left(p_{i}\right)}{g c p_{p_{i}}}-1} \overline{\mathcal{M}}_{p_{i}}^{k_{i}} \subseteq \mathbb{N}, \forall p_{i} \in P^{*} \\
\overline{\mathcal{M}}_{p_{i}}^{k_{i}}=k_{i} \cdot g c d_{p_{i}} .
\end{gathered}
$$

As a result, the number of partitions to be considered when searching for an optimal solution is reduced from $\Phi^{\prime}$ in Eq. (22) to $\Phi^{\prime \prime \prime}$ in Eq. (30).

\section{B. A more general optimization problem}

In many cases, it may be useful to introduce an additional criterion for Problem 1 so as to select, among all the solutions that provide the same optimal value of cycle time $\chi(\boldsymbol{M})$, those that also minimize the total weighted sum of tokens in the net. This problem has practical significance: under a given upper bound on the resources we aim to maximize the throughput and achieve this goal with a minimal cost.

Problem 2: Let $N$ be a TWMG and $\boldsymbol{y} \in \mathbb{N}^{|P|}$ be a cost vector as defined in Eq. (13). Given a positive real number $R$ that represents the upper bound on the cost of available resources and a small positive number $w \in \mathbb{R}^{+}$, we look for an initial marking $M$ which satisfies the following condition:

$$
\begin{aligned}
& \min \chi(\boldsymbol{M})+w \cdot \boldsymbol{y}^{T} \cdot \boldsymbol{M} \\
& \text { s.t. } \\
& \qquad \boldsymbol{y}^{T} \cdot \boldsymbol{M} \leq R
\end{aligned}
$$


Note that $w \in \mathbb{R}^{+}$should be sufficiently small so as to maintain the minimization of $\chi(\boldsymbol{M})$ as the prior requirement.

By substituting the objective function in Eq. (26) with the following function:

$$
\max \beta_{j}-w \cdot \boldsymbol{y}^{T} \cdot \boldsymbol{M}_{j},
$$

the optimal solution of Problem 2 can be found. Note that to solve Problem 2, we need to compute all the local optimal solutions and compare both the throughput and the cost of resources.

\section{EXPERIMENTAL RESULTS}

In this section, two types of experimental results are provided. Firstly, we test the proposed approach on a model of flexible manufacturing system (FMS) taken from the literature [29]. Second, a series of randomly generated nets are explored and the numerical results are given in Subsection VI-B. For the application of Algorithm 2, MATLAB has been used with the mixed ILPP toolbox YALMIP [43] on a Windows operating system with Pentium Dual-Core CPU $3.0 \mathrm{GHz}$ and $2 \mathrm{~GB}$ memory.

\section{A. Optimization of a flexible manufacturing system}

The TWMG model of an FMS [29] is shown in Fig. 8. This system is composed of three machines $\mathcal{U}_{1}, \mathcal{U}_{2}$ and $\mathcal{U}_{3}$. The manufacturing system is cyclic and can manufacture two products, denoted by $\mathcal{R}_{1}$ and $\mathcal{R}_{2}$. The production mix is $60 \%$ and $40 \%$ for $\mathcal{R}_{1}$ and $\mathcal{R}_{2}$, respectively. The production processes of these products are:

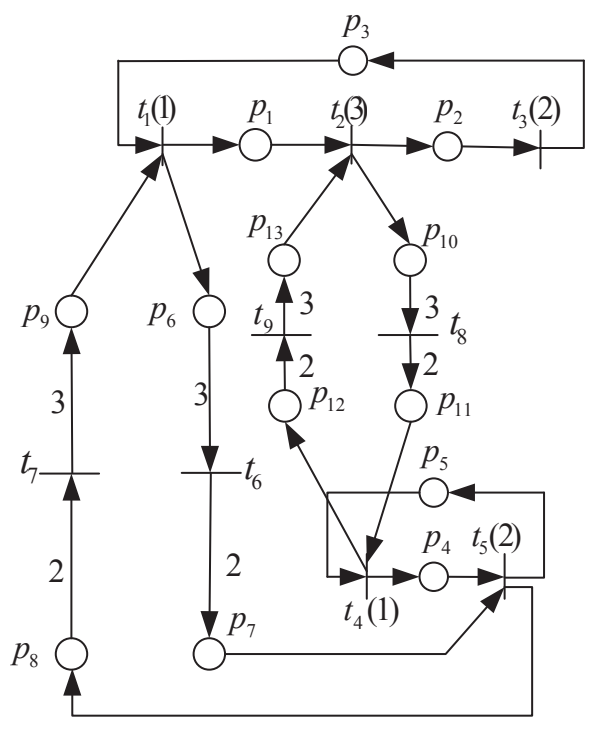

Fig. 8. The TWMG model of a flexible manufacturing system.

$$
\begin{cases}\mathcal{R}_{1}: & \left(\mathcal{U}_{1}, \mathcal{U}_{2}, \mathcal{U}_{3}\right) \\ \mathcal{R}_{2}: & \left(\mathcal{U}_{2}, \mathcal{U}_{1}\right)\end{cases}
$$


In this model, there are three types of elementary circuits:

- Process circuits: Model the manufacturing process. The tokens belonging to these circuits represent transportation resources.

- Command circuits: Model the control of the system. One command circuit is associated with each machine $\mathcal{U}_{1}$ and $\mathcal{U}_{2}$ to specify that they are cyclically used in both processes.

- Mixed circuits: These circuits are partially composed of parts of the command circuits and parts of the process circuits.

The PN model in Fig. 8 is a strongly connected TWMG with $n=|P|=13$ and $m=|T|=9$. There are six elementary circuits :

$$
\left\{\begin{array}{l}
\gamma_{1}=p_{1} t_{2} p_{2} t_{3} p_{3} t_{1} \\
\gamma_{2}=p_{4} t_{5} p_{5} t_{4} \\
\gamma_{3}=p_{10} t_{8} p_{11} t_{4} p_{12} t_{9} p_{13} t_{2} \\
\gamma_{4}=p_{6} t_{6} p_{7} t_{5} p_{8} t_{7} p_{9} t_{1} \\
\gamma_{5}=p_{2} t_{3} p_{3} t_{1} p_{6} t_{6} p_{7} t_{5} p_{5} t_{4} p_{12} t_{9} p_{13} t_{2} \\
\gamma_{6}=p_{10} t_{8} p_{11} t_{4} p_{4} t_{5} p_{8} t_{7} p_{9} t_{1} p_{1} t_{2}
\end{array}\right.
$$

where $\gamma_{1}$ and $\gamma_{2}$ are process circuits, $\gamma_{3}$ and $\gamma_{4}$ are command circuits, and $\gamma_{5}$ and $\gamma_{6}$ are mixed circuits.

The command circuits that model the control of the system must prevent two transitions corresponding to the same machine from being fired simultaneously. Then, for the command circuit $\gamma_{3}$ in Fig. 8, we assume that $M\left(p_{10}\right)=0$, $M\left(p_{11}\right)=0, M\left(p_{12}\right)=0$, and $M\left(p_{13}\right)=3$ and this command circuit cannot be allocated tokens any more.

The number of tokens in process circuits $\gamma_{1}$ and $\gamma_{2}$ represents that of available pallets for products, i.e., work in process. Thus, the cost of the resources in these circuits are the main economic consumption of the FMS. Tokens belonging to command circuits $\gamma_{3}$ and $\gamma_{4}$ represent information. We have $\lambda_{1}=10, \lambda_{2}=10, \lambda_{3}=1, \lambda_{4}=1$, $\lambda_{5}=1$, and $\lambda_{6}=1$.

The P-semiflow is $\boldsymbol{y}=\sum_{\gamma \in \Gamma} \lambda_{\gamma} \cdot \boldsymbol{y}_{\gamma}=(12,12,12,13,13,4,6,6,4,4,6,6,4)^{T}$ and the minimal T-semiflow is $\boldsymbol{x}=(3,3,3,2,2,1,1,1,1)^{T}$. We have $\phi_{1}=3, \phi_{2}=3, \phi_{3}=3, \phi_{4}=2, \phi_{5}=2, \phi_{6}=3, \phi_{7}=2, \phi_{8}=2, \phi_{9}=3$, $\phi_{10}=3, \phi_{11}=2, \phi_{12}=2, \phi_{13}=3, g c d_{p_{i}}=1(i=1, \ldots, 13)$ and $P^{*}=\left\{p_{2}, p_{6}, p_{8}, p_{10}, p_{12}\right\}$. Let us consider the following optimization problem:

$$
\begin{aligned}
& \min \chi(\boldsymbol{M})+w \cdot \boldsymbol{y}^{T} \cdot \boldsymbol{M} \\
& \text { s.t. } \\
& \left\{\begin{array}{l}
\boldsymbol{y}^{T} \cdot \boldsymbol{M} \leq 100 \\
M\left(p_{13}\right)=3, \\
M\left(p_{i}\right)=0, i=10,11,12
\end{array}\right.
\end{aligned}
$$

Now, the form represented by Eq. (26) can be immediately formulated. The number of variables is equal to 31 
and the number of constrains is equal to 90 . The markings of the TWMG are partitioned into $\Phi^{\prime}=3888$ subsets. According to Proposition 9 proposed in Section V-A, the number of partitions can be reduced to $\Phi^{\prime \prime \prime}=1296$.

We assume that the available money that can be used to purchase the resource is 100, i.e., $R=100$ and choose $w=10^{-8}$. By using Algorithm 2, we find an optimal solution $\boldsymbol{M}=(2,0,0,1,1,0,1,1,0,0,0,0,3)^{T}$ and $\chi(\boldsymbol{M})=11$ by considering the number of reduced partition $\Phi^{\prime \prime \prime}$. It implies that the actual usage of money is 74 and the cost of pallets is 50 .

\section{B. Test of random nets}

In this subsection, we present some numerical results for Problems 1 and 2 in Table II. All the tested nets are randomly generated under the assumption that each circuit has at least two places and at most six places and the weight of each arc (resp. delay of each transition) is a random integer number picked up from the interval $[1,6]$ (resp. $[1,10]$ ). For each of them we initialize the available resource $R$ to a positive number which is much bigger than $R^{*}$. We mention that if $R$ is a number that closes to $R^{*}$, the complexity of Problem 1 will be the same of Problem 2. Using the number of reduced partitions $\Phi^{\prime \prime \prime}$, we obtain the optimal solutions for Problems 1 and 2. For all the cases in Table II, we consider the cardinalities of $P$ and $T$ (net size), the number of partition $\Phi^{\prime}$, the number of reduced partition $\Phi^{\prime \prime \prime}$, the throughput $\beta$, the cost of resources $y^{T} \cdot \boldsymbol{M}$, and the CPU time for Problems 1 and 2. Note that "o.o.t" (out of time) in Table II means that the solution of net 6 for Problem 2 cannot be found within 48 hours.

As one can see, the solutions for Problem 1 can be obtained within a very short time. Once we compute an initial marking which makes the system reach its upper bound of the throughput, the algorithm will stop. Nevertheless, for Problem 2 which maximizes the throughput and minimizes the cost of resources, we need to explore all the local optimal solutions and compare both the throughput and the cost of resources. Among all the solutions that provide the same optimal value of throughput, we also want to reduce the cost of resources as much as possible. As a consequence, the computational time will be significantly longer than that of Problem 1 . When the net size becomes larger, we cannot obtain a solution of Problem 2 within a reasonable time. However, the model represented by a TWMG is much smaller than that generated with a TMG. In practical examples, the net size of a TWMG is quite reasonable thanks to the weight.

\section{CONCLUSION}

This paper deals with the cycle time optimization problem of deterministic TWMGs. The problem consists in finding an initial marking to minimize the cycle time while the weighted sum of tokens in places is less than or equal to a given value. To the best of our knowledge, this problem has not been addressed in the literature. We transform a TWMG into several equivalent TMGs and formulate a mixed integer linear programming problem solution from the study in [12] to compute an optimal initial marking. The conversion of the obtained marking for the equivalent TMG to a marking associated with the TWMG is presented. Some techniques are introduced to 
TABLE II

COMPUTATION RESULTS FOR PROBLEMS 1 AND 2 IN TERMS OF DIFFERENT INPUT NETS.

\begin{tabular}{|c|c|c|c|c|c|c|c|c|c|c|}
\hline \multicolumn{2}{|c|}{} & \multicolumn{3}{|c|}{ Problem 1 } & \multicolumn{3}{c|}{ Problem 2} \\
\hline $\mathrm{N}$ & $|P|$ & $|T|$ & $\begin{array}{c}\text { Nb. of } \\
\text { partitions } \Phi^{\prime}\end{array}$ & $\begin{array}{c}\text { Nb. of } \\
\text { reduced partitions } \Phi^{\prime \prime \prime}\end{array}$ & $\begin{array}{c}\text { Throughput } \\
\beta\end{array}$ & $\begin{array}{c}\text { Cost of resources } \\
y^{T} \cdot M\end{array}$ & $\begin{array}{c}\text { CPU } \\
\text { time }\end{array}$ & $\begin{array}{c}\text { Throughput } \\
\beta\end{array}$ & $\begin{array}{c}\text { Cost of resources } \\
y^{T} \cdot M\end{array}$ & $\begin{array}{c}\text { CPU } \\
\text { time }\end{array}$ \\
\hline 1 & 5 & 4 & 128 & 32 & 0.0476 & 24 & $4 \mathrm{~s}$ & 0.0476 & 16 & $41 \mathrm{~s}$ \\
\hline 2 & 8 & 7 & 4,608 & 384 & 0.0278 & 104 & $7 \mathrm{~s}$ & 0.0278 & 26 & $20 \mathrm{~min}$ \\
\hline 3 & 13 & 9 & 3,888 & 1,296 & 0.0909 & 212 & $15 \mathrm{~s}$ & 0.0909 & 74 & $1 \mathrm{~h}$ \\
\hline 4 & 17 & 14 & 73,728 & 1,536 & 0.0139 & 128 & $20 \mathrm{~s}$ & 0.0139 & 42 & $1.9 \mathrm{~h}$ \\
\hline 5 & 27 & 22 & $10,077,696$ & 20,736 & 0.025 & 134 & $30 \mathrm{~s}$ & 0.025 & 46 & $27 \mathrm{~h}$ \\
\hline 6 & 35 & 29 & $5.4 \mathrm{e}+11$ & 65,536 & 0.0069 & 196 & $42 \mathrm{~s}$ & o.o.t. & o.o.t. & o.o.t. \\
\hline
\end{tabular}

reduce the computational burden of computing the solution. It is shown that, in some cases, we do not need to enumerate all the possible structures to find the optimal solution.

More general allocation problems have been studied in the second part of the paper: among all the solutions that provide the same optimal value of throughput, we aim to obtain the one that also minimizes the cost of resources. The proposed method can also guarantee the convergence to the optimum.

Future work will aim to reduce the computational cost of the cycle time optimization problem by considering a subset of places. From a practical point of view, it may be interesting to put resources such as pallets and machines in some specific places instead of taking all places into consideration. The second perspective of our work is to study the cycle time optimization problem of TWMGs under infinite server semantics which is a more general case.

\section{ACKNOWLEDGEMENT}

This work was supported by the National Natural Science Foundation of China under Grant Nos. 61374068 and 61472295, the Recruitment Program of Global Experts, the Science and Technology Development Fund, MSAR, under Grant No. 078/2015/A3, and the Fundamental Research Funds for the Central Universities under Grant No. JB142001-15, Xidian University.

\section{REFERENCES}

[1] Z. W. Li, H. S. Hu, and A. R. Wang. "Design of liveness-enforcing supervisors for flexible manufacturing systems using Petri nets," IEEE Trans. Syst. Man Cybern. Part C, vol. 37, no. 4, pp. 517-526, 2007.

[2] Z. W. Li and M. Zhao. "On controllability of dependent siphons for deadlock prevention in generalized Petri nets," IEEE Trans. Syst. Man Cybern. Part A, vol. 38, no. 2, pp. 369-384, 2008.

[3] E. Teruel, P. Chrzastowski-Wachtel, J. M. Colom, and M. Silva. “On weighted T-Systems," Appl. Theory Petri Nets, vol. 616, pp. 348-367, 1992.

[4] P. Chrzastowski-Wachtel and M. Raczunas. "Orbits, half-frozen tokens and the liveness of weighted circuits," Workshop struct. concurrency theory, pp. 116-128, Springer Verlag, 1995.

[5] J. F. Zhang, M. Khalgui, Z. W. Li, G. Frey, O. Mosbahi, and H. B. Salah. "Reconfigurable coordination of distributed discrete event control systems," IEEE Trans. Control Syst. Technol., vol. 23, no. 1, pp. 323-330, 2015. 
[6] J. Campos, G. Chiola, and M. Silva. "Ergodicity and throughput bounds of Petri nets with unique consistent firing count vector," IEEE Trans. Software Eng., vol. 17, no. 2, pp. 117-125, 1991.

[7] A. Munier. "Régime asymptotique optimal d'un graphe d'événements temporisé généralisé: Application à un problème d'assemblage," RAIPO-APII, vol. 27, pp. 487-513, 1992.

[8] M. Nakamura and M. Silva. "Cycle time computation in deterministically timed weighted marked graphs," in Proc. 7th IEEE Int. Conf. Emerg. Technol. Factory Autom., vol. 2, pp. 1037-1046, 1999.

[9] M. Pocci, I. Demongodin, N. Giambiasi, and A. Giua. "Synchronizing sequences on a class of unbounded systems using synchronized Petri nets," Discrete Event Dyn. Syst., vol. 26, no. 1, pp. 85-108, 2016.

[10] A. Benabid-Najjar, C. Hanen, O. Marchetti, and A. Munier. "Periodic schedules for bounded timed weighted event graphs," IEEE Trans. Autom. Control, vol. 57, no. 5, pp. 1222-1232, 2012.

[11] N. Q. Wu, M. C. Zhou, and Z. W. Li. "Short-term scheduling of crude-oil operations: Petri net-based control-theoretic approach," IEEE Rob. Autom. Mag., vol. 22, no. 2, pp. 64-76, 2015.

[12] A. Giua, A. Piccaluga, and C. Seatzu. "Firing rate optimization of cyclic timed event graphs," Automatica, vol. 38, no. 1, pp. 91-103, 2002.

[13] Z. Y. Ma, Z. W. Li, and A. Giua. "Design of optimal Petri net controllers for disjunctive generalized mutual exclusion constraints," IEEE Trans. Autom. Control, vol. 60, no. 7, pp. 1774-1785, 2015.

[14] J. H. Ye, Z. W. Li, and A. Giua. "Decentralized supervision of Petri nets with a coordinator," IEEE Trans. Syst. Man Cybern. Syst., vol. 45, no. 6, pp. 955-966, 2015.

[15] F. Baccelli, G. Cohen, G. J. Olsder, and J. P. Quadrat. Synchronization and Linearity: An Algebra for Discrete Event Systems. New York, USA: Wiley, 1992.

[16] G. Cohen, S. Gaubert, and J. P. Quadrat. "Timed-event graphs with multipliers and homogeneous min-plus systems," IEEE Trans. Autom. Control, vol. 43, no. 9, pp. 1296-1302, 1998.

[17] J. Komenda, S. Lahaye, and J. L. Boimond. "Determinization of timed Petri nets behaviors," Discrete Event Dyn. Syst., pp. 1-25, 2015.

[18] P. Declerck. "Compromise approach for predictive control of timed event graphs with specifications defined by P-time event graphs," Discrete Event Dyn. Syst., to be published. doi:10.1007/s10626-016-0227-4.

[19] W. M. Boussahel, S. Amari, and R. Kara. "Analytic evaluation of the cycle time on networked conflicting timed event graphs in the (Max,+) algebra," Discrete Event Dyn. Syst., to be published, doi:10.1007/s10626-015-0220-3.

[20] B. Trouillet and J. C. Gentina. "On the linearization of weighted T-systems," Int. J. Prod. Res., vol. 46, no. 19, pp. 5417-5426, 2008.

[21] S. Hamaci, J. L. Boimond, S. Lahaye, and M. Mostefaoui. "On the linearizability of discrete timed event graphs with multipliers using (min,+) algebra," in Proc. 7th Workshop Discrete Event Syst., Reims, France, pp. 367-372, 2004.

[22] A. Benfekir, S. Hamaci, J. L. Boimond, and K. Labadi. "Performance evaluation of nonlinear weighted T-system," Int. J. Syst. Sci., vol. 44, no. 10, pp. 1948-1955, 2013.

[23] H. Wang and Q. Zeng. "Modeling and analysis for workflow constrained by resources and nondetermined time: An approach based on Petri nets," IEEE Trans. Syst., Man, Cybern. A, Syst., vol. 38, no. 4, pp. 802-817, 2008.

[24] K. V. Hee, H. Reijers, E. Verbeek, and L. Zerguini. "On the optimal allocation of resources in stochastic workflow nets," in Proc. 7th UK Perform. Eng. Workshop, pp. 23-34, UK, 2001.

[25] R. Li and S. Reveliotis. "Performance optimization for a class of generalized stochastic Petri nets," Discrete Event Dyn. Syst., vol. 25, no. 3, pp. 387-417, 2015.

[26] Y. L. Chen, P. Y. Hsu, and Y. B. Chang. "A Petri net approach to support resource assignment in project management," IEEE Trans. Syst., Man, Cybern. A, Syst., vol. 38, no. 3, pp. 564-574, 2008.

[27] R. J. Rodriguez, J. Julvez, and J. Merseguer. "On the performance estimation and resource optimization in process Petri nets," IEEE Trans. Syst., Man, Cybern. Syst., vol. 43, no. 6, pp. 1385-1398, 2013.

[28] M. Benazouz, O. Marchetti, A. Munier, and U. Pascal. "A new approach for minimizing buffer capacities with throughput constraint for embedded system design," in Proc. IEEE Int. Conf. Comp. Syst. Appl., Paris, France, May 2010.

[29] N. Sauer. "Marking optimization of weighted marked graphs," Discrete Event Dyn. Syst., vol. 13, no. 3, pp. 245-262, 2003.

[30] Z. He, Z. W. Li, and A. Giua. "Marking optimization of deterministic timed weighted marked graphs," in Proc. 10th IEEE Int. Conf. Autom. Sci. Eng., pp. 413-418, Taipei, Taiwan, August 2014. 
[31] Z. He, Z. W. Li, and A. Giua. "Optimization of deterministic timed weighted marked graphs," IEEE Trans. Autom. Sci. Eng., to be published, doi:10.1109/TASE.2015.2490538.

[32] Z. He, Z. W. Li, and A. Giua. "Cycle time optimization of deterministic timed weighted marked graphs," in Proc. 11th IEEE Int. Conf. Autom. Sci. Eng., pp. 274-279, Gothenburg, Sweden, August, 2015.

[33] J. Campos, G. Chiola, J. M. Colom, and M. Silva. "Properties and performance bounds for timed marked graphs," IEEE Trans. Fundam. Theory Appl., vol. 39, no. 5, pp. 386-401, 1992.

[34] T. Murata. "Petri nets: Properties, analysis and applications," Proc. IEEE, vol. 77, no. 4, pp. 541-580, 1989.

[35] A. J. Carlier and P. Chrtienne. Problème d'ordonnancement: Modélisation, complexité, algorithmes, Masson, Paris, 1988.

[36] J. V. Millo and R. De Simone. "Periodic scheduling of marked graphs using balanced binary words," Theor. Comput. Sci., vol. 458, pp. 113-130, 2012.

[37] A. Ghamarian, M. Geilen, S. Stuijk, T. Basten, A. Moonen, M. Bekooij, B. Theelen, and M. Mousavi. "Throughput analysis of synchronous data flow graphs," in Proc. 6th IEEE Int. Conf. Appl. Concurrency Syst. Design, pp. 25-36, June 2006.

[38] J. Campos, J. M. Colom, H. Jungnitz, and M. Silva. "Approximate throughput computation of stochastic marked graphs," IEEE Trans. Software Eng., vol. 20, no. 7, pp. 526-535, 1994.

[39] D. Y. Chao, M. C. Zhou, and D. T. Wang. "Multiple weighted marked graphs," in Proc. 12th IFAC Triennial World Congress, vol. 4, pp. 259-263, 1993.

[40] F. Sessego, A. Giua, and C. Seatzu. "HYPENS: a Matlab tool for timed discrete, continuous and hybrid Petri nets," in Proc. Int. Conf. Appl. Theor. Petri Nets, pp. 419-428, 2008.

[41] O. Marchetti and A. Munier. "A sufficient condition for the liveness of weighted event graphs," Eur. J. Oper. Res., vol. 197, no. 2, pp. 532-540, 2009.

[42] P. Chrzastowski-Wachtel and M. Raczunas. "Liveness of weighted circuits and the diophantine problem of frobenius," Fund. Comput. Theory, vol. 710, pp. 171-180, 1993.

[43] J. Löfberg. "A toolbox for modeling and optimization in MATLAB," in Proc. IEEE Int. symp. Comput.-Aided Control Syst. Des., pp. 284-289, Taipei, Taiwan, 2004. 